\title{
An improved LINMAP for multicriteria decision: designing customized incentive portfolios in an organization
}

\author{
Jessica Rubiano-Moreno ${ }^{1}$. Samuel Nucamendi-Guillén ${ }^{2}$ [1] \\ Alvaro Cordero-Franco ${ }^{3}$. Alejandro Rodríguez-Magaña ${ }^{4}$
}

Received: 30 December 2020 / Revised: 17 December 2021 / Accepted: 28 January 2022 /

Published online: 25 February 2022

(c) The Author(s) 2022

\begin{abstract}
This study proposes three new versions of the well-known linear programming technique for multidimensional preference analysis (LINMAP). LINMAP addresses the multi-criteria decision problem by analyzing individual differences in preferences in relation to a set of prespecified incentives in multidimensional attribute space. The proposed models satisfy the decision-maker's specific needs, such as determining a fixed number of incentives to be active or assigning a minimum/maximum weight for the active incentives. The performance of the developed models is assessed using information from a case study in which a decision-maker desires to determine an optimal portfolio of incentives based on the preferences of individuals surveyed. Experimental results confirm that the proposed models could obtain solutions according to the decision-maker's needs, yielding a better selection of incentives to activate and their corresponding distribution of the weights than those of the original LINMAP model. Moreover, the consistency of the proposed models is evaluated by performing a sensitivity analysis over database variations of the case study and comparing the outcomes with the results provided in the original case study. Overall, this work is promising when creating a design portfolio, considering individuals' different preferences.
\end{abstract}

Keywords Data analysis - Incentive $\cdot$ LINMAP $\cdot$ Mixed integer linear programming $\cdot$ Multicriteria decision making 


\section{Introduction}

Decision theory is concerned with the reasoning underlying an agent's decisions. For effective decision-making, it is essential to understand the market's preferences or behavior (or a specific segment of it) toward a specific set of attributes to design strategies, products, or services with high impact and profitability, which is known as multi-criteria decision analysis (Keeney and Raiffa 1976; Belton and Stewart 2002; Tzeng and Huang 2011; Greco et al. 2016). However, with a growing number of independent variables and increasing uncertainty, the complexity of decisions increases and the reliability of our intuitive judgments declines (Julmi 2019). Most decision activities involve evaluating multiple alternatives in a set of decision criteria, where these criteria are expected to conflict, i.e., an improvement in one may indicate a deterioration in another. Therefore, reliable methods and tools are required to support the choice between alternative courses of action (Cinelli et al. 2020).

This research is inspired by a study conducted on a group of university students (undergraduate and graduate) belonging to the millennial generation to identify their preferences over specific monetary and nonmonetary incentives from a predefined incentive portfolio. The study becomes relevant since this generation is currently economically active and, as mentioned in Ertas (2015), millennials have observed a volatile employment behavior, which translates into a high employee turnover rate. Thus, employers desire to identify the workers' motivations and design a portfolio of incentives that increase employee retention (permanence of workers in the organization). Moreover, Linder (2016) establishes that incentives motivate workers and, therefore, they can be included as a variable part of the salary to reward a better than required performance (Bateman and Ludwig 2003).

An organization that adequately incentivizes its workers and finds a balance between the purchasing power of its employees and the brand value manages to produce effectively and generates a sustainable organizational culture over time (Milne 2007). Organizations should incentivize employees whose performances are better than expected (Bandiera et al. 2009). Linder (2016) also highlighted the relevance of choosing the most appropriate incentives for organizations, which directly influence the employees' autonomous strategic actions. Contrariwise, if incentives set by an employer are perceived as devious, the effect will be counterproductive and will negatively affect workers' performance.

Currently, incentive portfolios within organizations are often designed according to the workers' positions or work areas (Gavish et al. 2000; Russo et al. 2018). This form of incentive assumes that different workers in the same position have the same social, economic, and family situation, neglecting that intrinsic and extrinsic motivations affect the most significant incentives (Sproten and Schwieren 2015; Yousaf et al. 2015). Therefore, determining a worker behavior model that allows identifying his/her preferences becomes crucial for managing human talent in organizations (Lai and Ishizaka 2020). An approach to study the latter situation is through multicriteria decision-making mathematical models (Barron and Barrett 1996). Moreover, identifying attribute weights is crucial in 
developing multiattribute models for selecting the best course of action (Horsky and Nelson 2006). This identification helps to design a product or service that meets expectations, aspirations, and specific requirements.

The linear programming technique for multidimensional analysis of preference (LINMAP) is a well-known searching method for identifying an optimal solution that minimizes the individual differences in preference judgments with regard to a set of stimuli (Srinivasan and Shocker 1973). By employing constrained least-absolute-differences, LINMAP estimates the coordinates of an ideal point considering the decision-maker's preferences. The objective is to appropriately quantify the preference level expressed by a worker or a group of workers toward specific attributes, characteristics, or incentives. Notably, after implementing the basic LINMAP, we observed that the original version does not necessarily reward the best-rated incentives accordingly (as it will be shown in Sect. 4), so the proposed improvement is devoted to helping the decision-maker by using a method to reward the incentives best rated by the individuals while minimizing of the total discrepancy among individual scores.

Therefore, in this study, we propose modified versions of LINMAP to solve a case study. These tailored versions are relevant in situations where the agent desires to compare a reference individual (a specific type of profile) with individuals participating in the survey. We also propose a biobjective function of the basic LINMAP formulation. In addition to the compromise solution, higher weights to the bestrated attributes are considered. Moreover, we develop new constraints to facilitate the decision-making process, for instance, when a minimum/maximum number of active incentives is desired.

Further, it can be used when the agent desires that the active incentives are associated with a minimum/maximum weight. The proposed biobjective function is evaluated as a mono-objective function via the weighted sum method to facilitate the solution process. Three versions of LINMAP are developed according to the requirements of the decision-maker. Finally, a case study was used to evaluate the performance of the versions, presenting the results of each model. The suitability of the proposed models is verified by examples of customized incentive portfolios based on the preferences toward a specific group of incentives.

The remainder of this paper is organized as follows. Section 2 presents a literature review on multicriteria decision models. Section 3 provides the formulations of modified LINMAP considering different criteria of the decision-maker. Section 4 presents the results of the computational experience over the case study. Finally, Sect. 5 discusses significant findings and suggests future research directions.

\section{Literature review}

In this section, relevant approaches regarding multicriteria decision models and preference functions are discussed. First, the closely related multicriteria decision models are described. Then, related approaches that inspired the development of the biobjective function preference function are presented. 


\subsection{Related multicriteria decision models}

Methods for measuring individual preferences toward a product/service can be broadly divided into two: compositional and decompositional.

In compositional methods, each component of the attitude or preference toward a given stimulus is evaluated separately. The global score is achieved by an appropriate combination of individual preferences. The method entails directly asking the subject about the importance of each attribute (classical scales). Meanwhile, decompositional methods typically begin by evaluating the stimulus and then explaining the differences due to inferred components of attitudes (Holbrook 1981).

Although compositional methods are considered unrealistic according to stereotyped responses and irrelevant attributes (El Gibari et al. 2019), they have attracted enormous research attention due to their simplicity in both the calculations and required analysis methods, such as the analytical hierarchy process (AHP, Saaty 1977 1977) and linear model for multidimensional preference analysis (LINMAP). In this sense, LINMAP emerges as a method that treats ordinal-scaled preference data as such when establishing individual-level criterion weights without requiring evaluating decision-makers' judgments separately or implementing comparison mechanisms that, in some cases, would also require to compute additional estimators (Horsky and Nelson 2006). For this reason, this study proposes variants to satisfy the decision-maker's specific needs, such as determining a fixed number of incentives to activate or assigning a minimum/maximum weight for the active incentives.

\subsection{Linear programming technique for multidimensional analysis of preference (LINMAP)}

The LINMAP methodology is based on a linear model for multidimensional analysis of preferences proposed by Srinivasan and Shocker (1973). LINMAP presents similarities with extensions of the UTA method that incorporate constraints to represent the intensity of agents' preferences (importance coefficients) so that the decision-maker can prioritize incentives in which the firm should invest since they are the ones that provide the most significant benefit to employees. However, this model quantitatively weighs attributes to be analyzed to rank them according to the preferences. According to Srinivasan and Shocker (1973), it is reasonable to assume that different dimensions must be weighted differently. LINMAP is particularly useful since it requires an ordinal scale for variables. It has been used in different areas when working with multicriteria decision-making approaches (Zhang and Lu 2006; Bereketli et al. 2011. Some adjustments have been made to the original model and applied to other fields (Mullet and Karson 1986; Xia et al. 2006; Li 2008; Tang and Hsu 2018).

Various studies have analyzed the performance of LINMAP, e.g., Pekelman and Sen (1974), Khairullah and Zionts (1979), Wittink and Cattin (1981). In these studies, real and simulated experiments were performed, where the estimations obtained by LINMAP, ANOVA, LOGIT, MONANOVA, and OLS models were compared. 
The LINMAP showed consistency in its results when there was a dominant attribute (Horsky and Rao 1984).

Horsky and Rao (1984) generalized LINMAP with the LINMAC model, which showed an improved performance in terms of consistency, uniqueness, and efficiency. In these experiments, the traditional LINMAP did not exhibit the best results, justifying the adjustments made to improve the outcomes. Velazquez et al. (2010) analyzed multiattribute models based on LINMAP in which scales and weights were combined. They concluded that using the $L_{\infty}$ scale was the most convenient.

Rasouli et al. (2011) integrated robust optimization to LINMAP, and it was applied to the European Foundation's quality management model. Kashef et al. (2018) combined LINMAP and the passive and active compensability multicriteria analysis models (PACMAN, Giarlotta 1998), which showed improved performance in terms of consistency, uniqueness, and certainty in solutions obtained in real problems.

In this study, modified versions of the traditional LINMAP are used to analyze individual differences between evaluations. A set of attributes related to incentives an organization currently offers or is willing to add to its incentive portfolio is considered. Each attribute corresponds to an incentive (monetary or nonmonetary). Each individual selects a level of importance (preference) according to an ipsative scale. Three versions of the model with different approaches are presented. The proposed models are applied to a case study, and the results are compared with those of the original LINMAP model (Srinivasan and Shocker 1973).

\subsection{Preference functions}

In conjoint analysis, an individual preference for a continuous attribute is often estimated for several discrete levels of the attribute using a part-worth function model (Cattin and Punj 1984). Commonly, for each attribute $j$, the preference of individual $i$ is registered by a discrete parameter $\left(C_{i j}\right)$, rated according to a numerical scale ranging from 1 to $K$, where 1 and $K$ represent the minimum and maximum rating, respectively. It is worth mentioning that these ratings are given on an ordinal scale. In order to use these values $C_{i j}$ in the LINMAP model, we need to change these ordinal preferences to numerical values $a_{i j}$, keeping the order structure. However, ordinal judgments cannot be arbitrarily transformed into a purely cardinal scale by means of a linear transformation. This clearly violates the character of the original (ordinal) data while imposing the same distances between neighboring ranks.

Abastante et al. (2019) proposed a parsimonious AHP methodology that allows dealing with decision problems involving seven or more alternatives effectively (reducing the amount of information handled by the decision-maker but preserving the soundness of the prioritization process), and in which the judgments provided by the individuals can be expressed using an ordinal scale.

Their methodology operates in five steps: (i) evaluation of the alternatives, (ii) definition of reference objects (done by the decision-maker), (iii) pairwise comparison of the reference objects by using Analytical Hierarchy Process (AHP), (iv) confirmation of the consistency of the pairwise comparisons with respect to the 
reference objects and (v) interpolation of the values obtained by AHP in step (iii) to get normalized evaluations of all objects [see Abastante et al. (2019) for details]. The main benefits of this methodology are: (1) Avoids the distortion of comparing more relevant objects with less relevant ones and (2) avoids rank reversal problems. The authors validated the effectiveness of their proposal by showing that the new parsimonious AHP outperformed the traditional AHP method in both consistency of the weights obtained (reducing the value of the mean standard error) and reducing calculations.

In this study, the parsimonious AHP method is implemented to estimate the utility functions $\left(a_{i j}\right)$ that will represent the level of preference of the surveyed individuals. To transform these utilities as required by LINMAP (to the scale of [0-1]), we performed a final normalization procedure that re-scale the previously obtained weights. These normalized utilities will be referred as $\alpha_{i}$. Depending on the decision-maker (DM) classification preferences, the maximum rating may correspond to the highest or the lowest value. On this scale, the lowest scale rating will have a normalized preference value of 0 , while the highest one will have a normalized preference value of 1 . On the other hand, when the maximum rating value is scored as 1 and the minimum one is denoted with the value of 5 , a readjustment must be made before. This readjustment is done as: $K+1-k_{i}$, where $k_{i}$ represents the $i$ th value of the scale. Moreover, the ratings of the individuals are also re-scaled using the expression $K-C_{i j}+1$, where $K$ denotes the lowest rating (with the highest magnitude), and $C_{i j}$ represents the rating provided by the individual. Therefore, the utility of the attribute which rating equals 1 (lowest) is 0 , and the utility for the highest rating is 1 . In other words, on an ipsative scale of 1 to 5 , the utility value of 1 is represented as 0 , and the one of 5 corresponds to 1 .

Finally, these normalized utilities $\left(\alpha_{i}\right)$ are used to determine the corresponding utility values for the selection made by the $i$ th individual to the $j$ th attribute $\left(a_{i j}\right)$. For instance, when the $i$ th individual rates the five attributes with the scores of 5, 4, 1,2 , and 3 , their corresponding utility functions will be $a_{i 1}=\alpha_{5}, a_{i 2}=\alpha_{4}, a_{i 3}=\alpha_{1}$, $a_{i 4}=\alpha_{2}, a_{i 3}=\alpha_{3}$.

The following section presents how the proposed adaptations are incorporated into the LINMAP method. The aim is to improve its performance when estimating the weights of the attributes and selecting those that represent stronger preferences.

\section{Proposed models}

This section presents the mathematical formulations developed to address the considered problem. In addition, the management implications and benefits (advantages) of each modification are described. Finally, a brief discussion of possible disadvantages that the proposed models may face is presented.

Formally, the problem under study can be formally defined as follows. There is a set $N$ of attributes to rate and a set $I$ of individuals who evaluate them. The numbers of attributes and individuals are denoted by $n$ and $m$, respectively. Each attribute is rated according to a numerical scale ranging from 1 to $k$, where 1 and $k$ represent the minimum and maximum rating, respectively. These ratings can be considered in an 
ordinal scale adjusted to a level of appropriate scale preference (in the range $[0,1]$ ). The proposed biobjective function finds the weights of attributes near the ideal solution (the best compromise solution), associating higher weights with the better-rated attributes.

The remaining parameters are as follows:

- $a_{i j}=$ Utility value (in percentage) of the score given by individual $i$ with respect to the attribute $j$.

- $\delta=$ Minimum difference (percentage) allowed between the utilities of individuals $i$ and $k$ for the attribute $j$.

In addition, the decision variables involved in the models are as follows:

- $w_{j}=$ Coefficient of importance (weight) assigned to attribute $j$.

- $z_{i k}=$ Value of discrepancy (adjustment) between the preferences of individuals $i$ and $k$ to reach the minimum allowed difference $\delta$.

\subsection{Mathematical formulation}

Based on a model proposed by Srinivasan and Shocker (1973), in which a monoobjective function was considered, we develop several variants to the formulation according to the specific requirements of the company.

The first modification involves a reformulation of the objective function in which we add a component that strongly rewards the best-rated attributes by individuals. Accordingly, we propose a function that balances the minimization of the total value of the discrepancy generated by the inconsistency in preferences for different attributes given by individuals and the prioritization of the best-rated attributes. Consequently, a new objective function that minimizes the difference between maximum utility and preferences established by individuals (in terms of utility) is proposed as follows:

$$
\text { Minimize : }\left\{\sum_{i=1}^{|I|} \sum_{k=1}^{|I|} z_{i k}, \sum_{j=1}^{|N|} w_{j} \sum_{i=1}^{|I|}\left(1-a_{i j}\right)\right\}
$$

The difference $1-a_{i j}$ determines the deviation from the upper bound that the rating chosen by an individual $i$ places for attribute $j$ (the highest possible value). The smaller the difference, the higher the rating provided by the individual. These differences are estimated for each individual and attribute. It is obvious that these difference values remain within the range $[0,1]$. The values associated with these differences are sum-weighted using the weights of each attribute in the original model. Accordingly, the attributes associated with small differences are rewarded. Then, they are summed up for each attribute and multiplied by their corresponding weight. Finally, these weighted sums are added up to estimate the global difference.

To reduce the complexity of the formulation, an artificial individual called "reference individual" is included. The medians associated with each attribute are 
considered the ratings placed by the reference individual, who will be denoted with the index 0 and included in the data file to estimate the difference with other individuals. This is motivated by the fact that the median represents the minimum rating for $50 \%$ of individuals and is used to compare individuals. When the median value is higher than the rate given by individual $i$, at least half of the individuals rated the incentive as equal to or greater than that of individual $i$. Otherwise, the rate of individual $i$ is greater than or equal to those of half of the individuals, which reduces the number of direct comparisons among customers. The proposed biobjective function is shown in Eq.(2).

$$
\text { Minimize : }\left\{\sum_{k=1}^{|I|} z_{0 k}, \sum_{j=1}^{|N|} w_{j} \sum_{k=1}^{|I|}\left(1-a_{k j}\right)\right\}
$$

Although both objectives are measured in the same units, they do not necessarily follow the same scale, i.e., they may have different orders of magnitude. For instance, the first objective may produce values between 1 and 5, whereas the second one may be between 0 and 1. According to Gunantara (2018), this situation causes two main issues. First, a bias in finding a tradeoff solution; second, complications arise when the resulting model is nonconvex.

To address this potential problem, a normalization procedure is applied to each objective. Normalization is crucial in ensuring consistent solutions according to the preferences expressed by the decision-maker (Mausser 2006). Therefore, we propose the following normalization (details are provided in the "Appendix"). For the first objective, we have:

$$
\text { Minimize }: \frac{\sum_{k=1}^{|I|} z_{0 k}}{Q_{\max }^{1}}
$$

For the second objective, we have:

$$
\text { Minimize }: \frac{\sum_{j=1}^{|N|} w_{j} \sum_{k=1}^{|I|}\left(1-a_{k j}\right)}{Q_{\max }^{2}}
$$

where $Q_{\text {max }}^{1}$ and $Q_{\text {max }}^{2}$ are the maximum values that can be obtained in each objective function and are used for standardizing objective values (having them in the same units). In this case, $Q_{\max }^{1}$, represents the maximum sum (over the incentives) of the positive discrepancies between the median and the score provided by the individuals. With respect to $Q_{\max }^{2}$, it denotes the maximum sum (over the incentives) of the differences between the highest possible score and the median score of the group (see the "Appendix" section for details).

After normalization, both objectives are in the range $[0,1]$. Exploiting the normalized objectives, we propose to use the weighted value method, which evaluates the proposed problem using a mono-objective function (Marler and Arora 2005). This approach uses a factor $\alpha \in(0,1]$ representing the percentage of importance assigned to the original objective, whereas its complement $(1-\alpha)$ 
denotes the percentage assigned to the other component. Therefore, the new objective function is expressed as follows:

$$
\text { Minimize }: \alpha \frac{\sum_{k=1}^{|I|} z_{0 k}}{Q_{\max }^{1}}+(1-\alpha) \frac{\sum_{j=1}^{|N|} w_{j} \sum_{k=1}^{|I|}\left(1-a_{k j}\right)}{Q_{\max }^{2}}
$$

It is important to note that if $\alpha=1$, then the objective function is similar to the one proposed by Srinivasan and Shocker (1973), whereas, if $\alpha \approx 0$, the best-rated attributes are considered primarily (regardless of the discrepancy factor).

According to the set of constraints, Eq. (6) determines the weight of each attribute by comparing the differences between the preferences of each pair of individuals (or comparing with a reference value) and assigning weights to meet a maximum difference allowed $\delta$ using the decision variable $z_{0 k}$.

$$
\sum_{j=1}^{|N|}\left(a_{0 j}-a_{k j}\right) w_{j}+z_{0 k} \geq \delta \quad \forall k \in\{1,2, \ldots,|I|\}
$$

In Eq. (6), $a_{0 j}$ represents the median of attribute $j$ (integrated into the preference matrix). Considering the differences with respect to the median, the score of each individual is subtracted from this centrality measure (added as a reference individual in the dataset). Then, these differences are summed up per attribute and then multiplied by the corresponding weight of the attribute. However, this equation can be extended to analyze the differences over all pairs $(i, k)$, substituting the parameter $a_{0 j}$ by $a_{i j}$ and estimating discrepancies using variable $z_{i k}$. As observed, increasing the value of $\delta$ provokes that most incentives take the value of zero (or being discarded). If the decision-maker desires to reduce the number of incentives to activate, an alternative could be to increase the value of this parameter.

Equation (7) forces percentage weights to be distributed at $100 \%$.

$$
\sum_{j=1}^{|N|} w_{j}=1
$$

Finally, Eqs. (8)-(9) defines decision variables used in the model.

$$
\begin{aligned}
& w_{j} \geq 0 \quad \forall j \in\{1,2, \ldots,|N|\} \\
& z_{0 k} \geq 0 \quad \forall k \in\{1,2, \ldots,|I|\}
\end{aligned}
$$

In this model, Eqs. (5)-(9), the identification of active attributes is determined by assigned weight. In other words, attributes considered active are those that have a weight greater than 0 . This version of the model is called M1.

The second version of the model is proposed for cases when an organization establishes a limit regarding the maximum number of incentives considered in its portfolio, which can be useful when the decision-maker needs to increase the budget (in percentage) substantially. Even when M1 can be adjusted to limit the number of incentives to activate by increasing the value of delta, there is no 
certainty regarding the minimum required value to produce the desired outcome. Therefore, we propose adding a new constraint that controls the number of active attributes (either a lower or upper bound). First, a new binary variable $q_{j}$ is considered, indicating whether the incentive $j$ is selected (1), i.e., $w_{j}>0$, or not (0).

This variable aids identify active attributes regarding assigned weights, which is achieved using Eqs. (10) to (12). An appropriate value of $M$ is given.

$$
\begin{gathered}
q_{j} \geq w_{j} \quad \forall j \in\{1,2, \ldots,|N|\} \\
q_{j} \leq M w_{j} \quad \forall j \in\{1,2, \ldots,|N|\} \\
q_{j} \in\{0,1\} \quad \forall j \in\{1,2, \ldots,|N|\}
\end{gathered}
$$

Since the value of $w \rightarrow 0$, a sufficiently large value of $M$ needs to be set for Eq. (11). In our experiments, we set $M=N^{3}$ as the big $M$ value.

Now, to determine the number of attributes to activate (both minimum and maximum), Eqs. (13), (14) [in conjunction with Eq. (12)] are used:

$$
\begin{aligned}
& \sum_{j=1}^{|N|} q_{j} \geq L \\
& \sum_{j=1}^{|N|} q_{j} \leq U
\end{aligned}
$$

where Eqs. (13) and (14) establish a minimum (L) and a maximum (U) number of desirable attributes to activate, respectively. Therefore, the complete model for this second version is given by Eqs. (5)-(14). This second model is called M2.

Another variation of the original model is proposed for cases when the decision-maker considers only the attributes that have a minimum or maximum weight. This model is equivalent to the version of limiting the number of active incentives but, instead of defining a maximum number of incentives to activate, they are limited by the minimum or maximum weight ( $\beta$ or $\gamma$, respectively) defined by the agent. In this case, the third proposed model adds two constraints, given by Eqs. (15) and (16), for the minimum and maximum weights, respectively.

$$
\begin{aligned}
& w_{j} \geq \beta q_{j} \quad \forall j \in\{1,2, \ldots,|N|\} \\
& w_{j} \leq \gamma q_{j} \quad \forall j \in\{1,2, \ldots,|N|\}
\end{aligned}
$$

Equation (15) guarantees that in case incentive $j$ is activated, the weight assigned to it is at least the value of $\beta$. Similarly, Eq. (16) ensures that the active incentives do not exceed a maximum established weight. In this case, the complete model contemplates Eqs. (5) - (12) and replaced Eqs. (13) and (14) with Eqs. (15) and (16), respectively. This third proposed model is called M3. 
Table 1 Attributes rating

\begin{tabular}{lllllllllll}
\hline Individual & At 1 & At 2 & At 3 & At 4 & At 5 & At 6 & At 7 & At 8 & At 9 & At 10 \\
\hline Ind 1 & 3 & 2 & 1 & 4 & 5 & 7 & 10 & 9 & 6 & 8 \\
Ind 2 & 1 & 3 & 2 & 5 & 4 & 9 & 6 & 10 & 8 & 7 \\
Ind 3 & 5 & 3 & 1 & 2 & 4 & 7 & 8 & 6 & 10 & 9 \\
Ind 4 & 5 & 2 & 1 & 4 & 3 & 8 & 9 & 10 & 6 & 7 \\
Ind 5 & 4 & 1 & 3 & 2 & 5 & 10 & 9 & 8 & 7 & 6 \\
Median & 4 & 2 & 1 & 4 & 4 & 8 & 9 & 9 & 7 & 7 \\
\hline
\end{tabular}

The parameter $\alpha$ controls the level of importance (in percentage) given to each component of the objective function. For instance, a small value of $\alpha$ indicates that most of the importance in the objective function is given to the activation of the best-rated incentives. On the contrary, higher values prioritize activating incentives in which most individuals give a rate equal to the median of the group. Regarding the parameter $\delta$, it helps to control the number of incentives to activate implicitly. That is, considering the rates given for a specific incentive, small values of $\delta$ allow activating incentives in which the median of the group is equal or slightly superior to the low rates given by the individuals (data distribution is skewed left). However, higher values of $\delta$ force the model to activate the incentives in which the data distribution is skewed right (high values are more frequent).

The proposed modified versions are compared with the original version introduced by Srinivasan and Shocker (1973). Setting $\alpha=1$ in the objective function determines whether the new versions allow activating incentives relevant to the individuals' preferences. An illustrative example is presented next to explain how the proposed model works whereas a case study is presented in Sect. 4.1 for which the proposed models are employed and compared. Finally, the obtained results are presented.

\subsection{Illustrative example}

Consider an example in which the decision-maker (DM) ask five individuals to rate ten different attributes using an ordinal ranking scale from 1 to 10 , where 1 denotes the lowest value (less preferred) and 10 represents the highest one (most preferred). The individual results and the median of each criterion are presented in Table 1.

Results in Table 1 indicate a clear preference for the attributes 6 to 10, which are expected to be the ones having the highest medians. Secondly, from the scale $\{1-10\}$, the analyst fixes four reference values $\{1,4,7,10\}$. These values will be used to generate the pairwise comparison matrix. The resulting matrix is shown in Table 2.

Following the AHP procedure described in Abastante et al. (2019), the DM obtains the consistency ratio (CR) to determine if the pairwise comparisons are consistent enough. In this case, we got the value of $C R=0.05$, which indicates that the DM provided a concordant prioritization. Then, the DM determines the 
Table 2 Pairwise comparison matrix

\begin{tabular}{lllll}
\hline & 1 & 4 & 7 & 10 \\
\hline 1 & 1 & $1 / 3$ & $1 / 6$ & $1 / 9$ \\
4 & 3 & 1 & $1 / 3$ & $1 / 6$ \\
7 & 6 & 3 & 1 & $1 / 3$ \\
10 & 9 & 6 & 3 & 1 \\
\hline
\end{tabular}

evaluations $\left(\gamma_{i}\right)$ for this predefined values. The resulting utilities are: $\gamma_{1}=0.05$, $\gamma_{4}=0.11, \gamma_{7}=0.26, \gamma_{10}=0.59$. After this, the corresponding utilities for the remaining rates are calculated using the interpolation formula shown in Eq. (17).

$$
\gamma_{r_{j}}=\gamma_{s}+\frac{\gamma_{s+1}-\gamma_{s}}{r_{s+1}-r_{s}} *\left(r_{j}-\gamma_{s}\right) \quad \forall r_{j} \in\left[r_{s}, r_{s+1}\right]
$$

where: $\gamma_{s}$ : Denotes the utility of the immediate previous evaluated rate. $\gamma_{s+1}$ : Denotes the utility of the immediate after evaluated rate. $r_{s}$ : Denotes the value of the immediate previous evaluated rate. $r_{s+1}$ : Denotes the value of the immediate after evaluated rate.

For instance, the utility for the value of rate $=2\left(\gamma_{2}\right)$ is determined using the utilities estimated for the rates 1 and 4 as follows:

$$
\gamma_{2}=0.05+\frac{0.11-0.05}{4-1} *(2-0.05) \approx 0.07
$$

Therefore, the corresponding utilities for the remaining rates $\{3,5,6,8,9\}$ are, $\gamma_{3}=0.09, \gamma_{5}=0.16, \gamma_{6}=0.21, \gamma_{8}=0.37, \gamma_{9}=0.48$, respectively.

After this, we apply the normalization procedure. The normalized utilities $\left(\alpha_{i}\right)$ are obtained by using Eq. (18).

$$
\alpha_{i}=\frac{\gamma_{i}-\gamma_{\min }}{\gamma_{\max }-\gamma_{\min }}
$$

The resulting normalized values are: $\alpha_{1}=0, \alpha_{4}=0.11, \alpha_{7}=0.39, \alpha_{10}=1$. The corresponding utilities for the remaining ratings $\{2,3,5,6,8,9\}$ are $\alpha_{2}=0.04$, $\alpha_{3}=0.07, \alpha_{5}=0.21, \alpha_{6}=0.30, \alpha_{8}=0.60, \alpha_{9}=0.8$. The corresponding utilities for the rate provided by each individual $i$ to the attribute $j\left(a_{i j}\right)$ are shown in Table 3.

Next, for each attribute, differences between the median and each individual are estimated as $a_{0 j}-a_{i j}$, where $a_{0 j}$ represents the median rate for the $j$ th attribute and $a_{i j}$ indicates the rate provided by the individual $i$ for the $j$ th attribute. These differences are shown in Table 4.

From Table 4, it can be observed that attribute 4 is rated above the median only by individual 2, this is the reason why most of the differences are positive. Attributes 3 and 5 are rated by almost all individuals at the same rate. Therefore, the medians are equal to the most repeated values. For the case of attribute 3, only the individual 5 ranks it slightly higher. Furthermore, it is worth highlighting that 
Table 3 Utility functions for the attributes

\begin{tabular}{lllllllllll}
\hline Individual & At 1 & At 2 & At 3 & At 4 & At 5 & At 6 & At 7 & At 8 & At 9 & At 10 \\
\hline Ind 1 & 0.07 & 0.04 & 0.00 & 0.11 & 0.21 & 0.39 & 1.00 & 0.80 & 0.30 & 0.60 \\
Ind 2 & 0.00 & 0.07 & 0.04 & 0.21 & 0.11 & 0.80 & 0.30 & 1.00 & 0.60 & 0.39 \\
Ind 3 & 0.21 & 0.07 & 0.00 & 0.04 & 0.11 & 0.39 & 0.60 & 0.30 & 1.00 & 0.80 \\
Ind 4 & 0.21 & 0.04 & 0.00 & 0.11 & 0.07 & 0.60 & 0.80 & 1.00 & 0.30 & 0.39 \\
Ind 5 & 0.11 & 0.00 & 0.07 & 0.04 & 0.21 & 1.00 & 0.80 & 0.60 & 0.39 & 0.30 \\
Median & 0.11 & 0.04 & 0.00 & 0.11 & 0.11 & 0.60 & 0.80 & 0.80 & 0.39 & 0.39 \\
\hline
\end{tabular}

attributes 7 and 8 received the highest rates. In both attributes, it can be observed that 3 out of 5 individuals rate them with a value equal or above the median. In particular, for attribute 8 , two of them rated it above the median, in contrast to attribute 7 , rated above the median by only one individual. That would make attribute 8 more attractive than attribute 7 in practical terms, since there is a $20 \%$ of the group rated it higher.

The illustrative example is solved by considering Model 1 (M1) and a value of $\alpha=0.5$, giving equal importance to each component of the objective function. For the parameter $\delta$, we increased its value in steps of 0.1 . The results are displayed in Table 5.

As a result, it is remarkable that the model activates the two attributes with the highest median values (attributes 7 and 8). Moreover, when the value of $\delta \geq 0.5$, only one incentive is activated (attribute 7). In this case, the active attribute is the one that is ranked as the highest. The results of the computed discrepancies per individual $k(\forall k \in\{1,2,3,4,5\})$ are displayed in Table 6 .

As expected, for small values of $\delta$ only a few discrepancy variables $\left(z_{0 k}\right)$ take positive values. Moreover, as the value of $\delta$ increases, the number of variables that take positive values increases, and the values themselves increase as well. For instance, when $\delta=\{0.2,0.3\}$ only variable $z_{02}$ remains inactive.

Another aspect to note is that for values of $\delta \geq 0.4$ (where the model activates only one attribute), all discrepancy values $\left(z_{0 k}\right)$ are positive, and their magnitude increases proportionally to the increase in the value of $\delta$ (see last five rows in Table 6). This can be explained by the fact that, when the minimum number of active incentives is set and, to preserve feasibility in the constraints (see Eq.(6)), the model activates always the same discrepancy variables and increases their values linearly with respect to the value of $\delta$. For this reason all the variables $z_{0 k}$ take positive values, but the total weight is assigned to only one incentive. However, the latter does not happen in the case study, where only some of them remained active. In other words, for the case study, the discrepancy variables activated at the lowest value of $\delta$ (when only one incentive is selected) remained active for higher $\delta$ values.

Finally, when setting $\alpha=1$, it is observed that M1 reports identical results that those obtained with $\alpha=0.5$, except for the case when $\delta=0$ where the model also activates attributes 1,2 and 4 , but with the weights of $0.06,0.47$, and 0.22 , respectively. As a result, all of the variables $z_{i k}$ maintain the value of zero. Additionally, 
Table 4 Differences between the median and each individual

\begin{tabular}{lrrrrrrrrrr}
\hline Comparison & At 1 & At 2 & At 3 & At 4 & At 5 & At 6 & At 7 & At 8 & At 9 & At 10 \\
\hline median-ind 1 & 0.04 & 0.00 & 0.00 & 0.00 & -0.09 & 0.21 & -0.20 & 0.00 & 0.09 & -0.21 \\
median-ind 2 & 0.11 & -0.03 & -0.04 & -0.10 & 0.00 & -0.20 & 0.50 & -0.20 & -0.21 & 0.00 \\
median-ind 3 & -0.10 & -0.03 & 0.00 & 0.07 & 0.00 & 0.21 & 0.20 & 0.50 & -0.61 & -0.41 \\
median-ind 4 & -0.10 & 0.00 & 0.00 & 0.00 & 0.04 & 0.00 & 0.00 & -0.20 & 0.09 & 0.00 \\
median-ind 5 & 0.00 & 0.04 & -0.07 & 0.07 & -0.09 & -0.40 & 0.00 & 0.20 & 0.00 & 0.09 \\
\hline
\end{tabular}

Table 5 Summary of weights obtained for each attribute per value of $\delta$

\begin{tabular}{lllllllllll}
\hline$\delta$ Weight & At 1 & At 2 & At 3 & At 4 & At 5 & At 6 & At 7 & At 8 & At 9 & At 10 \\
\hline 0.00 & 0.00 & 0.00 & 0.00 & 0.00 & 0.00 & 0.12 & 0.29 & 0.25 & 0.34 & 0.00 \\
0.10 & 0.00 & 0.00 & 0.00 & 0.00 & 0.00 & 0.00 & 0.50 & 0.50 & 0.00 & 0.00 \\
0.20 & 0.00 & 0.00 & 0.00 & 0.00 & 0.00 & 0.00 & 0.57 & 0.43 & 0.00 & 0.00 \\
0.30 & 0.00 & 0.00 & 0.00 & 0.00 & 0.00 & 0.00 & 0.72 & 0.28 & 0.00 & 0.00 \\
0.40 & 0.00 & 0.00 & 0.00 & 0.00 & 0.00 & 0.00 & 0.86 & 0.14 & 0.00 & 0.00 \\
0.50 & 0.00 & 0.00 & 0.00 & 0.00 & 0.00 & 0.00 & 1.00 & 0.00 & 0.00 & 0.00 \\
0.60 & 0.00 & 0.00 & 0.00 & 0.00 & 0.00 & 0.00 & 1.00 & 0.00 & 0.00 & 0.00 \\
0.70 & 0.00 & 0.00 & 0.00 & 0.00 & 0.00 & 0.00 & 1.00 & 0.00 & 0.00 & 0.00 \\
0.80 & 0.00 & 0.00 & 0.00 & 0.00 & 0.00 & 0.00 & 1.00 & 0.00 & 0.00 & 0.00 \\
0.90 & 0.00 & 0.00 & 0.00 & 0.00 & 0.00 & 0.00 & 1.00 & 0.00 & 0.00 & 0.00 \\
\hline
\end{tabular}

Table 6 Values of discrepancies per value of $\delta$

\begin{tabular}{llrrrl}
\hline$\delta z_{0 k}$ & \multicolumn{1}{l}{$z_{01}$} & \multicolumn{1}{c}{$z_{02}$} & \multicolumn{1}{c}{$z_{03}$} & \multicolumn{1}{c}{$z_{04}$} & $z_{05}$ \\
\hline 0 & 0.00 & 0.00 & 0.00 & 0.02 & 0.00 \\
0.1 & 0.20 & -0.05 & -0.25 & 0.20 & 0.00 \\
0.2 & 0.31 & 0.00 & -0.13 & 0.29 & 0.11 \\
0.3 & 0.45 & 0.00 & 0.01 & 0.36 & 0.24 \\
0.4 & 0.57 & 0.00 & 0.16 & 0.43 & 0.37 \\
0.5 & 0.70 & 0.00 & 0.30 & 0.50 & 0.50 \\
0.6 & 0.80 & 0.10 & 0.40 & 0.60 & 0.60 \\
0.7 & 0.90 & 0.20 & 0.50 & 0.70 & 0.70 \\
0.8 & 1.00 & 0.30 & 0.60 & 0.80 & 0.80 \\
0.9 & 1.10 & 0.40 & 0.70 & 0.90 & 0.90 \\
\hline
\end{tabular}

when $0.1 \leq \delta \leq 0.4$ the number of active incentives decreases to two (attributes 4 and 7), reducing to 1 (attribute 7) when $\delta \geq 0.5$.

In practice, the DM can effectively implement this method at the moment of deciding how to distribute an increase in the budget for the current portfolio of incentives. In other words, the resulting weights will lead the company to determine which incentives are worth investing in and in which percentage. For instance, 
considering the illustrative example shown above: imagine that the DM is informed to distribute a certain amount of money among the most preferred attributes. The DM desires to fix only one attribute to reward with the total. After implementing the model, attribute seven must be selected. However, suppose the DM chooses to divide the budget between the top 4 preferred attributes. In that case, the DM has to distribute the available increase as follows: $12 \%$ for attribute $6,29 \%$ for attribute 7 , $28 \%$ for attribute 8 , and $34 \%$ for attribute 9 .

\section{Computational results}

\subsection{Case study}

To validate the effectiveness of the proposed models, a survey was conducted on a group of university students. The sampling was nonprobabilistic, considering two characteristics of students: (1) belonging to the millennial generation and (2) professional experience. Aspects related to socioeconomic status or the training level were not considered selection criteria to delimit the study's coverage. The survey evaluated the level of preference over 10 different incentives (monetary and nonmonetary). Ipsative responses were handled and ordered by type of incentive according to their preference. This study was conducted in Guadalajara, Jalisco, Mexico. After reviewing the quality of collected data, a sample of 188 individuals was obtained.

Most millennials sampled were between 21 and 25 years old; they represent $65.99 \%$ of the sample. The range from 26 to 30 years old constituted $22.84 \%$ of the sample, and the remaining $11.17 \%$ were over 31 years. Regarding gender, $53.81 \%$ and $46.19 \%$ of the sample were male and female, respectively. In terms of marital status, $87.31 \%$ and $12.69 \%$ of the sample were single and married, respectively. Further, $93.40 \%$ of the sample did not have children.

Concerning the socioeconomic level, the majority belonged to the middle-level stratum $(68.02 \%$ ), whereas $3.55 \%$ and $28.43 \%$ were in the low- and high-level strata, respectively. Regarding household composition, $67.01 \%$ lived with their families, $20.81 \%$ stayed alone or with friends, and only $12.18 \%$ lived in their own household. Accordingly, the majority had a relative or partner as their primary financial support (63.96\%), and the remainder were those who contributed to the household (36.04\%). Regarding the highest degree of education, the majority had a professional career (72.59\%), 26.86\% were postgraduate studies, and only $3.55 \%$ of the surveyed individuals marked high school as their highest degree. Considering income level, the majority (43.15\%) declared a salary between 5000 and 10,000 MXN (Mexican pesos), $23.86 \%$ reported income in a range of $10,001-20,000 \mathrm{MXN}, 17.77 \%$ were in a range of 20,001-30,000 MXN, and 15.25\% earned more than 30,000 MXN.

To analyze the performance of the models on the case study, five different values of $\alpha=\{0.1,0.3,0.5,0.7,0.9\}$ and ten values of $\delta=\{0,0.1,0.2,0.3,0.4,0.5,0.6,0.7,0.8,0.9\}$ were selected for computational experiments, since those parameters directly affect the number and type of incentives to activate. The parameter $\alpha$ affects the weight for each objective function whereas $\delta$ affects the number of active incentives, as this value increases the number of actives 
Table 7 Ranking of the incentives according to the median of the rates

\begin{tabular}{lll}
\hline Incentive & Median & Rank \\
\hline Career plan* & 6 & 4 \\
Christmas bonus & 4 & 6.5 \\
Flexible work schedule* & 8 & 1 \\
Health insurance* & 6 & 4 \\
Leisure zones at work* & 2 & 9 \\
Loans & 1 & 10 \\
Performance bonus & 7 & 2 \\
Scholarships* & 3 & 8 \\
Training* & 6 & 4 \\
Vacation premiums & 4 & 6.5 \\
\hline
\end{tabular}

* means that the incentive is non-monetary

incentives decreases. In addition, four reference rate values $r_{s}=\{1,4,5,7\}$ are considered to construct the pairwise comparison matrix that will be used to obtain the utilities to incorporate as input for the model. Recall that three versions of the model were proposed: M1, M2, and M3.

In the following subsections, the results of each version are presented and analyzed. To facilitate the discussion of the results, the rank of each incentive is displayed in Table 7. In the case of ties, the average rank is displayed.

From Table 7, the attribute with the highest median was ranked as 1, whereas the attribute with the lowest median was classified as 10 . In the case of ties, the mean rank was considered. This information was used to determine whether the proposed approach rewarded the incentives ranked at the top of the list. The symbol (*) indicates that the incentive is nonmonetary.

\subsection{Computational results considering M1}

The first analysis entails evaluating the results of the proposed model and preserving the original set of constraints proposed by Srinivasan and Shocker (1973). Figure 1 displays the summarized results. In addition, each subfigure shows the results for each value of $\alpha$ when considering all possible values of $\delta$.

In general, the model decreased the number of active incentives as the value of $\delta$ increased. In particular, when $\delta>0.7$, the model only activated one incentive, which occurred for all $\alpha$ values. In addition, all discrepancy values were maintained with the highest value of $\delta$. Concerning $\alpha$, the number of active incentives increased as the objective function converted into that of the original LINMAP model (i.e., when $\alpha \rightarrow 1$ ). As mentioned above, for high values of $\delta$, the number of comparisons that satisfied the difference value decreased. Thus, the number of active discrepancy variables $z_{0 k}$ increased. Therefore, the model tried minimizing these variables by fixing them to the values of $\delta$ or $1+\delta$ if possible. As such, the model minimized the number of active incentives to reach the minimum total discrepancy of the system. 


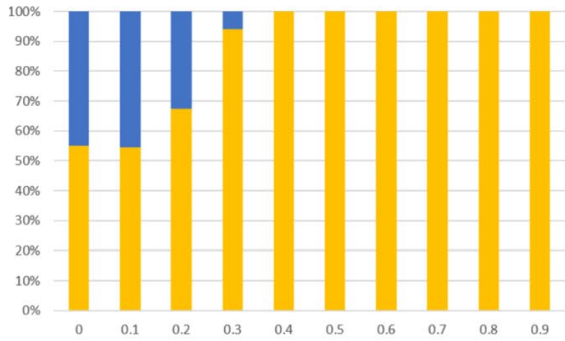

(a) $\alpha=0.1$

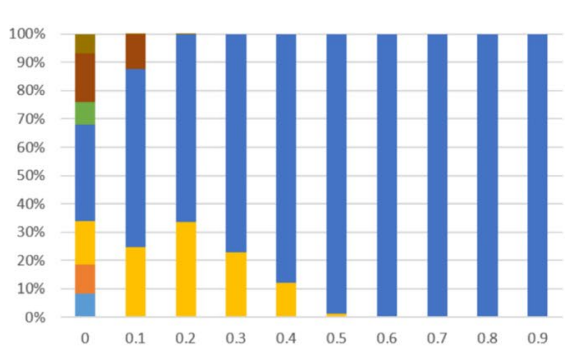

(c) $\alpha=0.5$

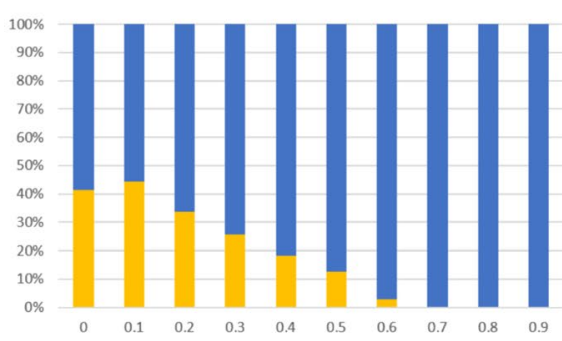

(b) $\alpha=0.3$

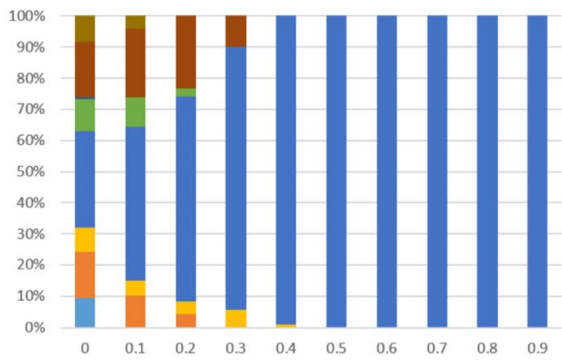

(d) $\alpha=0.7$

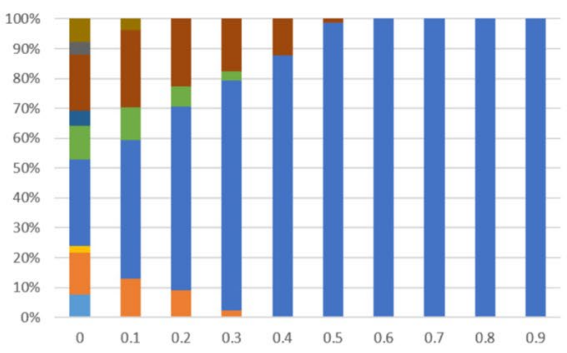

(e) $\alpha=0.9$

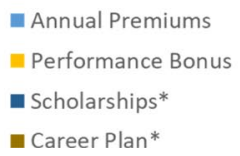

Christmas Bonus

Flexible Work Schedule*

- Training*
- Loans

- Health Insurance*

- Leisure zones at work*

Fig. 1 Results of model 1 (M1) segmented by value of $\alpha$

Another aspect to highlight is that for $\alpha \geq 0.5$, the model activated more than half of the available incentives (especially for small values of $\delta$ ). In other words, when having medium or high weights for the first objective, the model activated the incentives that minimize the discrepancy between the individual selection for those objectives, but they did not necessarily represent the incentives best-ranked by the majority. Moreover, when three active incentives were considered as the maximum number of incentives, the model preserved the same behavior as the basic one for $\delta \geq 0.3$. Hence, we could conclude that if the decision-maker desires to activate 


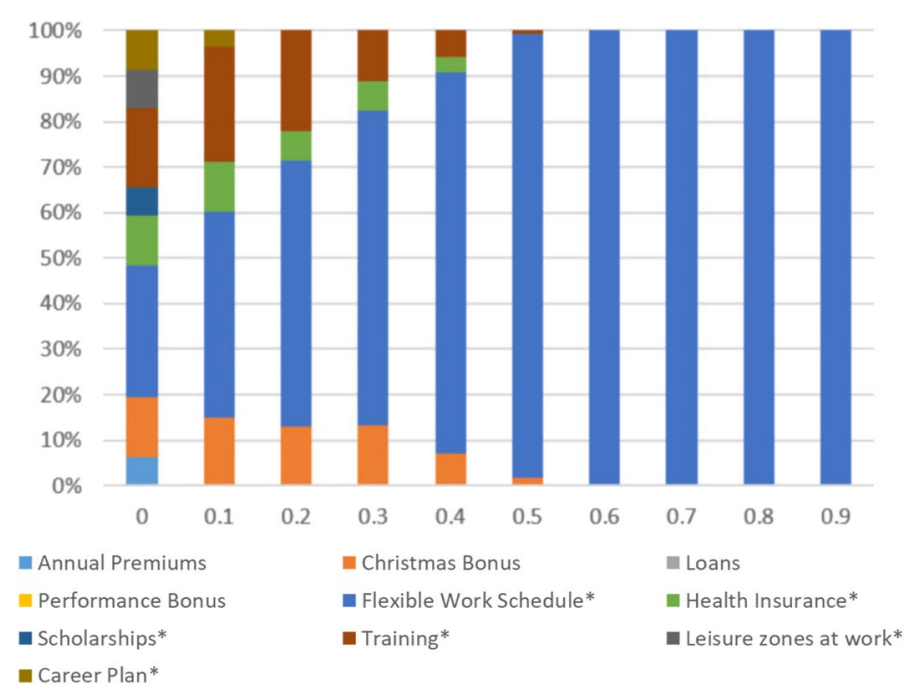

Fig. 2 Results of Model 1 (M1) considering $\alpha=1$ (original LINMAP model)

more than one incentive, then small values of $\delta$ and high values of $\alpha$ are suitable. However, this does not guarantee the activation of a specific number of incentives.

To complement the analysis, M1 was assessed with $\alpha=1$, i.e., only considering the first normalized objective function. The obtained results are illustrated in Fig. 2.

From the results shown in Figs. 1e and $2(\alpha \geq 0.9$ and $\delta \geq 0.1)$ the model activated the same incentives as when $\alpha=1$. The only exception was when $\alpha=1$ and $\delta=0$, where the model activated 9 of 10 available incentives (one more than when $\alpha=0.9$ and $\delta=0$ ). Hence, it could be confirmed that M1 reacted appropriately to different values of $\alpha$ and $\delta$.

To verify if the enhanced formulations M2 and M3 appropriately reacted to the agent's purposes, a similar analysis was performed.

\subsection{Computational results of $M 2$}

The second approach entailed evaluating the performance of the model when the decision-maker established a maximum number of incentives to activate. For the case study, a maximum number of three incentives was established $(U \leq 3)$, and the minimum number of incentives to activate was maintained as zero $L \geq 0$. Figure 3 illustrates the results.

M2 presents a similar behavior to M1. In general, as observed in Figs. 1 and 3 for $\alpha \leq 0.5$, both models report almost the same active incentives (except for the case when $\delta=0$ and $\alpha=0.5$ ). For $\alpha=\{0.7,0.9\}$, the results are similar for $\delta \geq 0.4$; when $\delta \leq 0.3 \mathrm{M} 2$ activates the three incentives with highest weights in M1.

As previously mentioned, M2 was evaluated by considering only the first normalized objective function $(\alpha=1)$ to investigate how the proposed constraints affected the solution of the model. Figure 4 illustrates the results. M1 and M2 

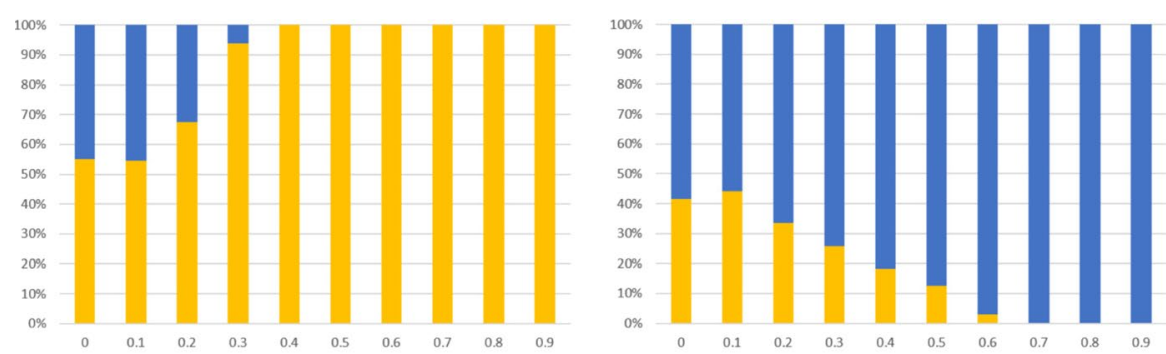

(a) $\alpha=0.1$

(b) $\alpha=0.3$
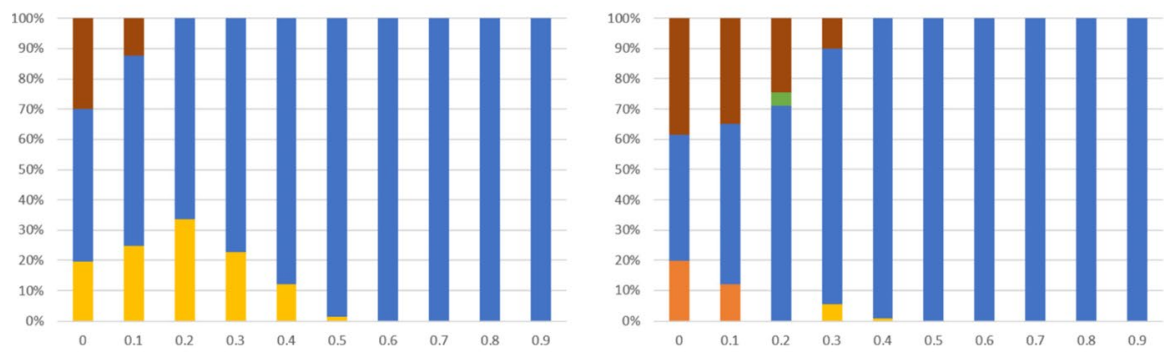

(c) $\alpha=0.5$

(d) $\alpha=0.7$

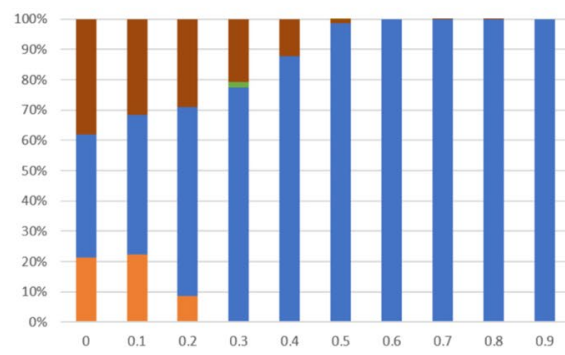

(e) $\alpha=0.9$

Annual Premiums
Performance Bonus
- Scholarships*
_ Career Plan*

- Christmas Bonus

Loans

Elexible Work Schedule*

- Training*

- Health Insurance*

- Leisure zones at work*

Fig. 3 Results of model 2 (M2) with a maximum of 3 active incentives

reported the same results for $\delta \geq 0.5$, whereas when $\delta \leq 0.4 \mathrm{M} 2$ activates the three incentives with highest weights in M1 (see Figs. 2, 4).

Another analysis arises when the agent desired to determine a maximum number of incentives to activate. However, instead of establishing an upper bound, a minimum desired weight was specified for the incentives worth activating. The results are reported in the following subsection. 


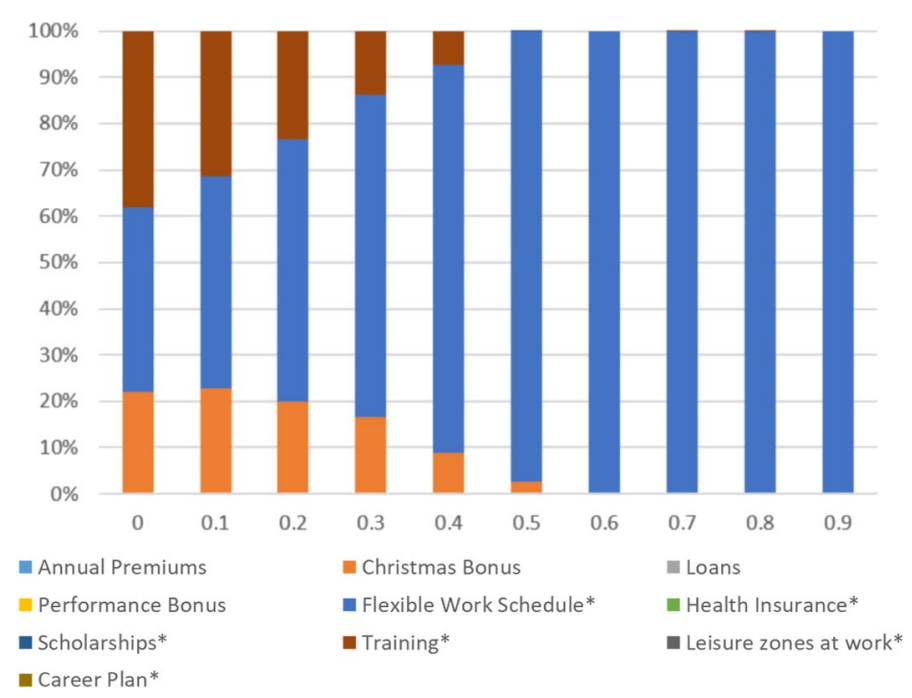

Fig. 4 Results of model 2 (M2) with a maximum of 3 active incentives considering $\alpha=1$

\subsection{Computational results of M3}

Recall that M3 is similar to M2, wherein a limited number of active incentives is considered. However, instead of that assumption, a minimum and maximum desired weights for the active incentives were established. In other words, the incentives to be activated were rewarded with at least the minimum weight and at most the maximum one. For the case study, $\beta=0.3$ and $\gamma=1$ were chosen. Figure 5 illustrates the results.

Based on the results shown in Fig. 5, when $\alpha=0.1$, solutions in M3 are similar in structure that the ones obtained in M2, but with weights within the established limits in Eqs. (15) and (16) (see Figs. 3a, 5a). It should be noted that when $\alpha=0.1$ and $\delta \geq 0.5$, the solution changes with respect to the ones obtained in M1 and M2, with Flexible work schedule as the only one active. When a solution in M2 contains an active incentive with a small weight, the solution in M3 eliminates that incentive, as can be seen when $\alpha=0.7$ and $\delta=0.2,0.3$.

A final analysis was conducted to validate the performance of LINMAP when the first normalized objective function was considered, $\alpha=1$. The results are shown in Fig. 6. Again, the structure of the solution remains between M2 and M3, in terms of active incentives, except in the cases where the solution contains lower weights active incentives in $\mathrm{M} 2(\delta \geq 0.2)$, see Figs. 4 and 6 .

In all previous results, increasing the value of $\delta$ reduces the number of activated incentives (as explained during the formulation of M1 and M2). However, M2 helped identify the most preferred incentives to activate when a predefined (maximum) number was desired since the beginning. Regarding M3, it helped establish the minimum percentage to assign to active incentives (proportional) with an increase in the original budget. 


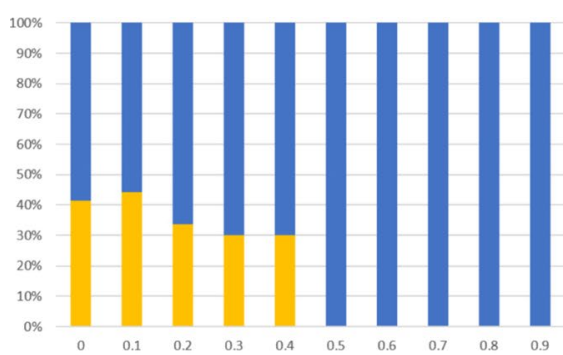

(a) $\alpha=0.1$

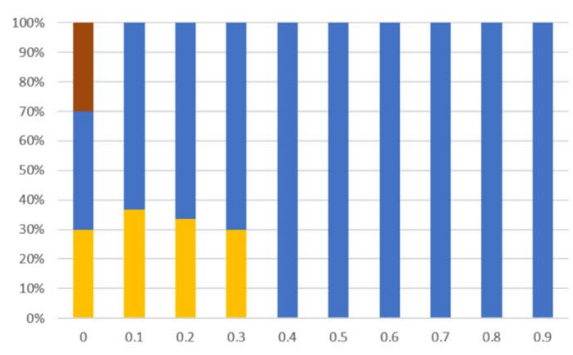

(c) $\alpha=0.5$

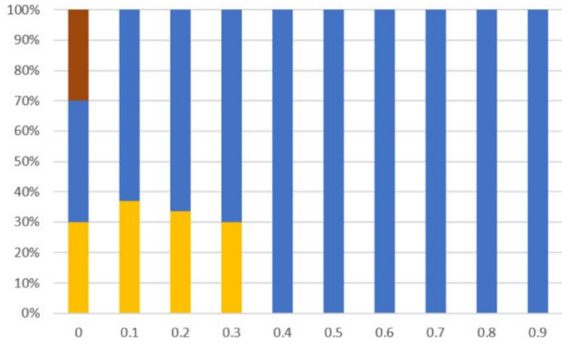

(b) $\alpha=0.3$

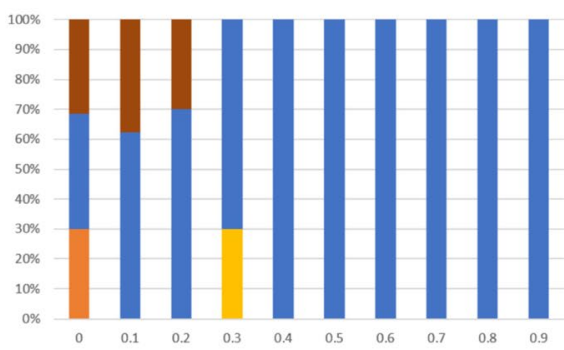

(d) $\alpha=0.7$

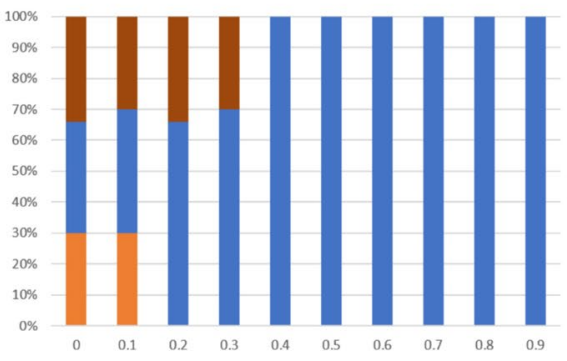

(e) $\alpha=0.9$

Annual Premiums

- Performance Bonus

- Scholarships*

- Career Plan*

\section{- Christmas Bonus}

- Flexible Work Schedule*

- Training*
- Loans

- Health Insurance*

- Leisure zones at work*

Fig. 5 Results of model 3 (M3) establishing $\beta=0.3$

From this analysis, it is evident that the proposed modified versions reward the best-ranked incentives for most individuals. However, the weights may vary when individual expectations or the population of surveyed individuals (sample size) is changed. Therefore, a complementary analysis is conducted by creating sub-scenarios to analyze how the models react when the database changes. This sensitivity analysis verifies the consistency of the proposed models. The following subsection reports the obtained results. 


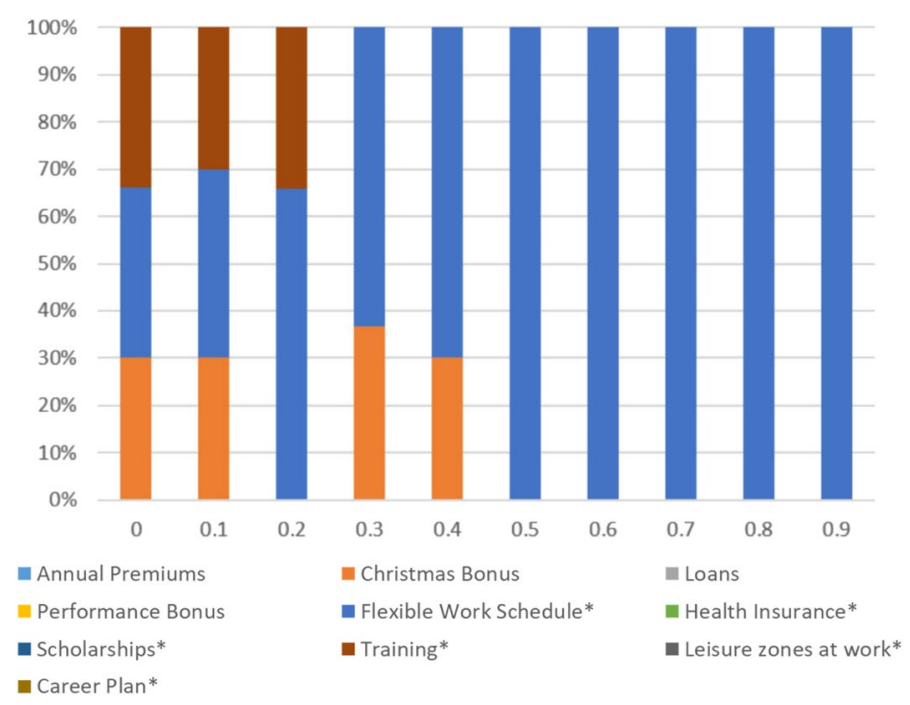

Fig. 6 Results of model 3 (M3) establishing $\beta=0.3$ and $\alpha=1$

Table 8 Summary of the weight for the incentives for the M1 model with $\alpha=0.1$

\begin{tabular}{|c|c|c|c|c|c|c|}
\hline \multirow{2}{*}{$\begin{array}{l}\alpha=0.1 \\
\text { Type of incentive }\end{array}$} & \multicolumn{3}{|c|}{$\delta=0$} & \multicolumn{3}{|c|}{$\delta=0.5$} \\
\hline & Min & Avg & $\operatorname{Max}$ & Min & Avg & $\operatorname{Max}$ \\
\hline Career plan* & 0.00 & 0.00 & 0.00 & 0.00 & 0.00 & 0.00 \\
\hline Christmas Bonus & 0.00 & 0.00 & 0.00 & 0.00 & 0.00 & 0.00 \\
\hline Flexible work schedule* & 0.00 & 0.35 & 0.45 & 0.00 & 0.00 & 0.00 \\
\hline Health insurance* & 0.00 & 0.00 & 0.00 & 0.00 & 0.00 & 0.00 \\
\hline Leisure zones at work* & 0.00 & 0.00 & 0.00 & 0.00 & 0.00 & 0.00 \\
\hline Loans & 0.00 & 0.00 & 0.00 & 0.00 & 0.00 & 0.00 \\
\hline Performance bonus & 0.55 & 0.65 & 1.00 & 1.00 & 1.00 & 1.00 \\
\hline Scholarships* & 0.00 & 0.00 & 0.00 & 0.00 & 0.00 & 0.00 \\
\hline Training* & 0.00 & 0.00 & 0.00 & 0.00 & 0.00 & 0.00 \\
\hline Annual premiums & 0.00 & 0.00 & 0.00 & 0.00 & 0.00 & 0.00 \\
\hline
\end{tabular}

* means that the incentive is non-monetary

\subsection{Sensitivity analysis}

To evaluate the robustness of the proposed models, a complementary analysis was conducted. Subs-cenarios were generated to analyze potential variations in the individuals considered in the case study. These sub-scenarios were generated by randomly selecting $90 \%$ of individuals from the original dataset. In total, five sub-scenarios were generated. Notably, the median was separately recalculated for each scenario to properly perform the experiment. All three model versions were implemented. 
Table 9 Summary of the weight for the incentives for the M1 model with $\alpha=0.5$

Table 10 Summary of the weight for the incentives for the M1 model with $\alpha=0.9$

Table 11 Summary of the weight for the incentives for the M2 model with $\alpha=0.1$

\begin{tabular}{|c|c|c|c|c|c|c|}
\hline \multirow{2}{*}{$\begin{array}{l}\alpha=0.5 \\
\text { Incentive }\end{array}$} & \multicolumn{3}{|l|}{$\delta=0$} & \multicolumn{3}{|c|}{$\delta=0.5$} \\
\hline & Min & Avg & Max & Min & Avg & $\operatorname{Max}$ \\
\hline Career plan* & 0.04 & 0.05 & 0.06 & 0.00 & 0.00 & 0.00 \\
\hline Christmas Bonus & 0.04 & 0.10 & 0.12 & 0.00 & 0.00 & 0.00 \\
\hline Flexible work schedule* & 0.36 & 0.38 & 0.40 & 0.99 & 0.99 & 0.99 \\
\hline Health insurance* & 0.00 & 0.01 & 0.03 & 0.00 & 0.00 & 0.00 \\
\hline Leisure zones at work* & 0.00 & 0.00 & 0.00 & 0.00 & 0.00 & 0.00 \\
\hline Loans & 0.00 & 0.00 & 0.00 & 0.00 & 0.00 & 0.00 \\
\hline Performance bonus & 0.15 & 0.19 & 0.21 & 0.01 & 0.01 & 0.01 \\
\hline Scholarships* & 0.00 & 0.00 & 0.00 & 0.00 & 0.00 & 0.00 \\
\hline Training* & 0.18 & 0.20 & 0.24 & 0.00 & 0.00 & 0.00 \\
\hline Annual premiums & 0.05 & 0.08 & 0.09 & 0.00 & 0.00 & 0.00 \\
\hline
\end{tabular}

* means that the incentive is non-monetary

\begin{tabular}{|c|c|c|c|c|c|c|}
\hline \multirow{2}{*}{$\begin{array}{l}\alpha=0.9 \\
\text { Incentive }\end{array}$} & \multicolumn{3}{|c|}{$\delta=0$} & \multicolumn{3}{|c|}{$\delta=0.5$} \\
\hline & Min & Avg & Max & Min & Avg & $\operatorname{Max}$ \\
\hline Career plan* & 0.04 & 0.05 & 0.06 & 0.00 & 0.00 & 0.00 \\
\hline Christmas Bonus & 0.14 & 0.17 & 0.19 & 0.00 & 0.01 & 0.03 \\
\hline Flexible work schedule* & 0.25 & 0.28 & 0.32 & 0.97 & 0.99 & 1.00 \\
\hline Health insurance* & 0.00 & 0.03 & 0.07 & 0.00 & 0.00 & 0.00 \\
\hline Leisure zones at work* & 0.00 & 0.08 & 0.21 & 0.00 & 0.00 & 0.00 \\
\hline Loans & 0.00 & 0.00 & 0.00 & 0.00 & 0.00 & 0.00 \\
\hline Performance bonus & 0.02 & 0.04 & 1.00 & 0.00 & 0.00 & 1.00 \\
\hline Scholarships* & 0.01 & 0.05 & 0.07 & 0.00 & 0.00 & 0.00 \\
\hline Training* & 0.22 & 0.23 & 0.25 & 0.00 & 0.00 & 0.01 \\
\hline Annual premiums & 0.03 & 0.07 & 0.09 & 0.00 & 0.00 & 0.00 \\
\hline
\end{tabular}

* means that the incentive is non-monetary

\begin{tabular}{|c|c|c|c|c|c|c|}
\hline \multirow{2}{*}{$\begin{array}{l}\alpha=0.1 \\
\text { Incentive }\end{array}$} & \multicolumn{3}{|c|}{$\delta=0$} & \multicolumn{3}{|c|}{$\delta=0.5$} \\
\hline & Min & Avg & $\operatorname{Max}$ & Min & Avg & $\operatorname{Max}$ \\
\hline Career plan* & 0.00 & 0.00 & 0.00 & 0.00 & 0.00 & 0.00 \\
\hline Christmas Bonus & 0.00 & 0.00 & 0.00 & 0.00 & 0.00 & 0.00 \\
\hline Flexible work schedule* & 0.00 & 0.35 & 0.45 & 0.00 & 0.00 & 0.00 \\
\hline Health insurance* & 0.00 & 0.00 & 0.00 & 0.00 & 0.00 & 0.00 \\
\hline Leisure zones at work* & 0.00 & 0.00 & 0.00 & 0.00 & 0.00 & 0.00 \\
\hline Loans & 0.00 & 0.00 & 0.00 & 0.00 & 0.00 & 0.00 \\
\hline Performance bonus & 0.55 & 0.65 & 1.00 & 1.00 & 1.00 & 1.00 \\
\hline Scholarships* & 0.00 & 0.00 & 0.00 & 0.00 & 0.00 & 0.00 \\
\hline Training* & 0.00 & 0.00 & 0.00 & 0.00 & 0.00 & 0.00 \\
\hline Annual premiums & 0.00 & 0.00 & 0.00 & 0.00 & 0.00 & 0.00 \\
\hline
\end{tabular}

* means that the incentive is non-monetary 
Table 12 Summary of the weight for the incentives for the M2 model with $\alpha=0.5$

Table 13 Summary of the weight for the incentives for the M2 model with $\alpha=0.9$

Table 14 Summary of the weight for the incentives for the M3 model with $\alpha=0.1$

\begin{tabular}{|c|c|c|c|c|c|c|}
\hline \multirow{2}{*}{$\begin{array}{l}\alpha=0.5 \\
\text { Incentive }\end{array}$} & \multicolumn{3}{|l|}{$\delta=0$} & \multicolumn{3}{|c|}{$\delta=0.5$} \\
\hline & Min & Avg & Max & Min & Avg & $\operatorname{Max}$ \\
\hline Career plan* & 0.00 & 0.00 & 0.00 & 0.00 & 0.00 & 0.00 \\
\hline Christmas Bonus & 0.00 & 0.00 & 0.00 & 0.00 & 0.00 & 0.00 \\
\hline Flexible work schedule* & 0.50 & 0.50 & 0.50 & 0.99 & 0.99 & 0.99 \\
\hline Health insurance* & 0.00 & 0.00 & 0.00 & 0.00 & 0.00 & 0.00 \\
\hline Leisure zones at work* & 0.00 & 0.00 & 0.00 & 0.00 & 0.00 & 0.00 \\
\hline Loans & 0.00 & 0.00 & 0.00 & 0.00 & 0.00 & 0.00 \\
\hline Performance bonus & 0.20 & 0.20 & 0.21 & 0.01 & 0.01 & 0.01 \\
\hline Scholarships* & 0.00 & 0.00 & 0.00 & 0.00 & 0.00 & 0.00 \\
\hline Training* & 0.28 & 0.30 & 0.30 & 0.00 & 0.00 & 0.00 \\
\hline Annual premiums & 0.00 & 0.00 & 0.00 & 0.00 & 0.00 & 0.00 \\
\hline
\end{tabular}

* means that the incentive is non-monetary

\begin{tabular}{llllllll}
\hline$\alpha=0.9$ & \multicolumn{1}{l}{$\delta=0$} & & & & $\delta=0.5$ & \\
\cline { 2 - 3 } \cline { 7 - 8 } Incentive & Min & Avg & Max & & Min & Avg & Max \\
\hline Career plan* & 0.00 & 0.00 & 0.00 & & 0.00 & 0.00 & 0.00 \\
Christmas bonus & 0.00 & 0.18 & 0.24 & & 0.00 & 0.01 & 0.03 \\
Flexible work schedule* & 0.37 & 0.39 & 0.40 & & 0.97 & 0.99 & 1.00 \\
Health insurance* & 0.00 & 0.00 & 0.00 & & 0.00 & 0.00 & 0.00 \\
Leisure zones at work* & 0.00 & 0.05 & 0.27 & 0.00 & 0.00 & 0.00 \\
Loans & 0.00 & 0.00 & 0.00 & 0.00 & 0.00 & 0.00 \\
Performance bonus & 0.00 & 0.00 & 0.00 & & 0.00 & 0.00 & 0.00 \\
Scholarships* & 0.00 & 0.00 & 0.00 & 0.00 & 0.00 & 0.00 \\
Training* & 0.37 & 0.38 & 0.40 & & 0.00 & 0.00 & 0.01 \\
Annual premiums & 0.00 & 0.00 & 0.00 & 0.00 & 0.00 & 0.00 \\
\hline
\end{tabular}

* means that the incentive is non-monetary

\begin{tabular}{|c|c|c|c|c|c|c|}
\hline \multirow{2}{*}{$\begin{array}{l}\alpha=0.1 \\
\text { Incentive }\end{array}$} & \multicolumn{3}{|c|}{$\delta=0$} & \multicolumn{3}{|c|}{$\delta=0.5$} \\
\hline & Min & Avg & $\operatorname{Max}$ & Min & Avg & $\operatorname{Max}$ \\
\hline Career plan* & 0.00 & 0.00 & 0.00 & 0.00 & 0.00 & 0.00 \\
\hline Christmas Bonus & 0.00 & 0.00 & 0.00 & 0.00 & 0.00 & 0.00 \\
\hline Flexible work schedule* & 0.00 & 0.35 & 0.45 & 0.00 & 0.00 & 0.00 \\
\hline Health insurance* & 0.00 & 0.00 & 0.00 & 0.00 & 0.00 & 0.00 \\
\hline Leisure zones at work* & 0.00 & 0.00 & 0.00 & 0.00 & 0.00 & 0.00 \\
\hline Loans & 0.00 & 0.00 & 0.00 & 0.00 & 0.00 & 0.00 \\
\hline Performance bonus & 0.55 & 0.65 & 1.00 & 1.00 & 1.00 & 1.00 \\
\hline Scholarships* & 0.00 & 0.00 & 0.00 & 0.00 & 0.00 & 0.00 \\
\hline Training* & 0.00 & 0.00 & 0.00 & 0.00 & 0.00 & 0.00 \\
\hline Annual premiums & 0.00 & 0.00 & 0.00 & 0.00 & 0.00 & 0.00 \\
\hline
\end{tabular}

* means that the incentive is non-monetary 
Table 15 Summary of the weight for the incentives for the M3 model with $\alpha=0.5$
Table 16 Summary of the weight for the incentives for the M3 model with $\alpha=0.9$

\begin{tabular}{|c|c|c|c|c|c|c|}
\hline \multirow{2}{*}{$\begin{array}{l}\alpha=0.5 \\
\text { Incentive }\end{array}$} & \multicolumn{3}{|c|}{$\delta=0$} & \multicolumn{3}{|c|}{$\delta=0.5$} \\
\hline & Min & Avg & $\operatorname{Max}$ & Min & Avg & $\operatorname{Max}$ \\
\hline Career plan* & 0.00 & 0.00 & 0.00 & 0.00 & 0.00 & 0.00 \\
\hline Christmas Bonus & 0.00 & 0.00 & 0.00 & 0.00 & 0.00 & 0.00 \\
\hline Flexible work schedule* & 0.50 & 0.50 & 0.50 & 0.99 & 0.99 & 0.99 \\
\hline Health insurance* & 0.00 & 0.00 & 0.00 & 0.00 & 0.00 & 0.00 \\
\hline Leisure zones at work* & 0.00 & 0.00 & 0.00 & 0.00 & 0.00 & 0.00 \\
\hline Loans & 0.00 & 0.00 & 0.00 & 0.00 & 0.00 & 0.00 \\
\hline Performance bonus & 0.20 & 0.20 & 0.21 & 0.01 & 0.01 & 0.01 \\
\hline Scholarships* & 0.00 & 0.00 & 0.00 & 0.00 & 0.00 & 0.00 \\
\hline Training* & 0.28 & 0.30 & 0.30 & 0.00 & 0.00 & 0.00 \\
\hline Annual premiums & 0.00 & 0.00 & 0.00 & 0.00 & 0.00 & 0.00 \\
\hline
\end{tabular}

* means that the incentive is non-monetary

\begin{tabular}{|c|c|c|c|c|c|c|}
\hline \multirow{2}{*}{$\begin{array}{l}\alpha=0.9 \\
\text { Incentive }\end{array}$} & \multicolumn{3}{|l|}{$\delta=0$} & \multicolumn{3}{|c|}{$\delta=0.5$} \\
\hline & Min & Avg & Max & Min & Avg & $\operatorname{Max}$ \\
\hline Career plan* & 0.00 & 0.00 & 0.00 & 0.00 & 0.00 & 0.00 \\
\hline Christmas Bonus & 0.00 & 0.18 & 0.24 & 0.00 & 0.01 & 0.03 \\
\hline Flexible work schedule* & 0.37 & 0.39 & 0.40 & 0.97 & 0.99 & 1.00 \\
\hline Health insurance* & 0.00 & 0.00 & 0.00 & 0.00 & 0.00 & 0.00 \\
\hline Leisure zones at work* & 0.00 & 0.05 & 0.27 & 0.00 & 0.00 & 0.00 \\
\hline Loans & 0.00 & 0.00 & 0.00 & 0.00 & 0.00 & 0.00 \\
\hline Performance bonus & 0.00 & 0.00 & 0.00 & 0.00 & 0.00 & 0.00 \\
\hline Scholarships* & 0.00 & 0.00 & 0.00 & 0.00 & 0.00 & 0.00 \\
\hline Training* & 0.37 & 0.38 & 0.40 & 0.00 & 0.00 & 0.01 \\
\hline Annual premiums & 0.00 & 0.00 & 0.00 & 0.00 & 0.00 & 0.00 \\
\hline
\end{tabular}

* means that the incentive is non-monetary

To facilitate the analysis, the extreme and middle values for $\delta$ and $\alpha$ were considered; i.e., $\delta=0,0.5$ and $\alpha=0.1,0.5,0.9$. Tables 8,9 and 10 summarize the results of the five sub-scenarios for M1; Tables 11, 12 and 13 summarize results for M2; Tables 14,15 and 16 summarize the results for M3. As in previous results, when $\delta$ was fixed to a large value, the models activated only one incentive. Therefore, the values $\delta \geq 0.6$ were excluded from the report.

For each table, the first column displays the name of the instance. Columns 2 and 5 report the minimum weight for each incentive. Columns 3 and 6 indicate the average weight per incentive; columns 4 and 7 report the maximum values for the weights of each incentive. All these values are summarized based on the values of $\delta$ for the five sub-scenarios.

From Tables 8, 9 and 10, it can be observed that M1 preserves the same structure of the solution presented in Fig. 1 ; if $\delta=0$ the Performance bonus, Flexible work 
schedule and Training become active incentives with higher weights. When $\delta=0.5$ and $\alpha=0.1$ the Performance bonus is the only incentive active as it can be observed in the Max columns of Table 8. Whereas if $\alpha=0.5,0.9$ Flexible work schedule is the one that is activated in most of the sub-scenarios.

Regarding M2, results presented in Tables 11, 12 and 13 results present a similar behaviour to the outcomes presented in Fig. 3. In the case of $\delta=0$ and $\alpha=0.1,0.5$ the M2 only activates Flexible work schedule, Performance bonus and Training as the most, whereas for $\alpha=0.9$ Flexible work schedule, Training, Christmas bonus and Leisure zones at work are active incentives. When $\delta=0.5$, the structure of the solution remains with only one active incentive, first Performance bonus for $\alpha=0.1$ and Flexible work schedule for $\alpha=0.5,0.9$.

Again, the sensitivity analysis for M3 presented in Tables 14 to 16 displays an identical behaviour as the M3 solutions presented in Fig. 5, which confirms that the model reacts appropriately to the variations in the scenarios when considering these kind of constraints. In particular, when $\alpha=\{0.1,0.5\}$, the results are identical with $\delta=\{0.5\}$. However, when $\alpha=\{0.9\}$ M3 discards the Performance bonus from the list of active incentives. For $\delta=0$ the weight of Performance bonus decreases as $\alpha$ increases; if $\alpha=0.1$ Flexible work schedule is active in the solution; whereas if $\alpha=0.5$ Training is also an active incentive; and for $\alpha=0.9$ Leisure zones at work and Christmas bonus are added to the active incentives and Performance bonus is not anymore in the solution.

In summary, the obtained results confirmed the effectiveness and consistency of the proposed versions of LINMAP. From the experiment, it can be concluded that the solution structure was consistent, but small changes appeared in the incentive weights (Tables $8,9,10,11,12,13,14,15,16$ ). Particularly, when $\alpha=0.1$, the solutions for M1, M2, and M3 had a similar structure. In general, for M1 and M3, the Performance bonus and Flexible work schedule remained active incentives (Tables 8 and 14). For M2 (Table 11), the model preserved the incentives of the Flexible work schedule and Performance bonus as active; depending on the subscenario, the model might activate any incentive belonging to the Career plan and Training (with weights less or equal to 0.2). Moreover, from Tables 8, 11, and 14, when $\delta=0.5$, the incentives of the Flexible work schedule and Performance bonus did not always remain active in the optimal solution (see column Min these tables). The latter implies that at least in one scenario one of the mentioned incentives is excluded, and its total weight is assigned to the other.

When $\alpha=0.5$, the models presented a mixed behavior. For M1, when $\delta=0$, the model preserved the assignment of the highest weight for the Flexible work schedule; however, depending on the scenario, some potential incentives could also be activated (Tables 9, 12, 15). For M2 and M3, the numbers of potential incentives to activate were reduced to 5 and 3 , respectively. Notably, when $\delta=0.5$, only the Flexible work schedule remained active (last three columns of the above tables).

Finally, when $\alpha=0.9$, the structure of the solution remained the same for all models in most cases. When $\delta=0$, the scholarship incentive, active in M1 (Table 10), was not activated for M2 and M3 (Tables 13, 16). For all models, the incentive of loans, the lowest ranked incentive, was not considered in any scenario. Moreover, the Flexible work schedule was the only active incentive for all of the 
models (Tables 10,13,16), which was a highly ranked incentive. Overall, in all scenarios, the models maintained active the same incentives as in the original experiments when considering $\alpha=\{0.1,0.5\}$. In general, as $\delta$ increased, the number of active incentives decreased. In particular, for most cases, when $\delta \geq 0.5$, only one incentive remained active in the optimal solution.

In summary, the results indicated that when the highest weight was assigned to the second objective function, the solution was oriented to activate the highestranked incentives: the flexible work schedule and performance bonus (preserving the highest weight for the nonmonetary one). Meanwhile, when higher weights were assigned to the first objective function, the solution sought incentives in which individuals gave a score close to the median value. Consequently, more incentives were activated. Remarkably, in these scenarios, the two highest-ranked incentives were preserved in the solution, accompanied by other incentives, such as training, health insurance, and career plan.

As final considerations, one aspect to observe was that for $\alpha \geq 0.5$, M1 and M3 activated the highest and lowest numbers of incentives, respectively. In addition, attributes unactivated for M1 remained inactive for M2 and M3.

After reviewing the sensitivity analysis (Cinelli et al. 2020), it became evident that the models were consistent. Moreover, the proposed models are easy to use since their processing times are minimal. Collecting data is a simple process that only requires the completion of a survey.

\subsection{Managerial insights}

The results presented for different scenarios indicated that the group of millennials surveyed in this study preferred nonmonetary incentives (Flexible work schedule, Training, and Career plan) to monetary ones (Performance bonus was the predominant economic incentive). Even when these incentives were activated with different weights (according to the different values of $\alpha$ and $\delta$ ), the hierarchy in which they appeared remained.

In addition, the findings of this study agree with existing results in the literature. For instance, Stein and Martin (2015) claimed that millennials prefer independence and flexibility in their work schedules. In our study, these incentives were always activated. The apparition of the performance bonus in numerous scenarios supported the results of Linder (2016) and Kosfeld et al. (2016), who found that people prefer monetary incentives depending on their work.

According to Kim (2018), this generation prefers short courses where they can learn punctual abilities instead of traditional graduate programs, which can justify the solutions of this study. In addition, the incentive of health insurance that appeared in some scenarios could be explained by the study of Nielsen (2015), which considered 60 countries, concluding that it is essential to include healthcare and medical as incentives. Furthermore, Stein and Martin (2015) investigated the relevance of gaining higher positions in one's work, whether it be in one's current employment or a different one, for this generation. 
Other incentives were not activated in any scenario. As an example, for loans, Kim (2018) concluded that millennials prefer using external credit organizations to those at their work. For instance, asking for a loan in their organization would conflict with their interest toward considering potential jobs in different organizations that could offer higher positions, which explains why the surveyed individuals ranked this incentive as the lowest.

Finally, as explained at the end of Sect. 3, this method can be helpful to discard the incentives that does not need to be supported, but also to quantify the level of preference for the incentives in which the company must invest in. For instance, if the firms desires to increase the available budget in 10,000 monetary units and desires to know how to distribute this increase among the most preferred attributes. In this case, the DM can distribute the money of increase according to the obtained weight of each incentive. For instance, considering the results of Table 16, the amount of money that can be assigned to Flexible work schedule can vary between 37 and $40 \%$ of the increase (around 3700 and 4000 monetary units). In the case of training, a similar assignment can be done, leaving the remaining percentage to invest in Leisure zones at work. For the incentives in which the weight was zero, it can be inferred that those incentives can remain as is.

\section{Conclusions}

In this study, the multicriteria decision problem for analyzing individual differences in preference judgments concerning a set of incentives prespecified in a multidimensional attribute space is investigated. For this problem, modified versions of LINMAP for multidimensional analysis of preferences were proposed. The versions added a second component in the objective function, which allowed prioritizing the selection of best-ranked attributes. Moreover, a parameter $\alpha$ was introduced to validate the effectiveness of the modified versions over the original LINMAP model. Finally, a sensitivity analysis was conducted to verify the robustness by varying the database for a case study.

Based on the outcomes, all proposed versions facilitated the decision-making process. Therefore, the modified LINMAP models consider the different requirements of practitioners, which can be deduced from the number and type of incentives selected during the experiments. In addition, the obtained results showed that the proposed models were robust to variations in the preference matrix. Decisionmakers can use the proposed models to obtain different incentive portfolios and select an ideal solution for their organizations. A possible disadvantage is that the use of the weighted sum method generates compensations in the objective function; i.e., an increase in the weight of the first objective value compensates for the loss of other objectives. This may be an interesting topic for future studies.

Future research directions can focus on determining new attributes to invest in instead of focusing on only identifying their percentage of preference for the current ones, considering all these while working within a limited budget. 
In addition, variations in individuals' decisions can be analyzed. Moreover, the assumption of a linear transformation of ordinal preferences imposes the same distance between neighboring ranks, which is not always sustained. Therefore, a LINMAP with ordinal preferences can be analyzed; its results may be compared with the three proposed versions, aiming to show discrepancies in the solution structure.

\section{Appendix: Objectives normalization procedure}

Proposition 1 The maximum of the objective functions (2), are given by:

$$
\begin{aligned}
& Q_{\text {max }}^{1}=\max _{l}\left\{\sum_{k=1}^{|I|} \max \left\{0, \delta-\left(a_{0 l}-a_{k l}\right)\right\}\right\} \\
& Q_{\text {max }}^{2}=\max _{l}\left\{\sum_{k=1}^{|I|}\left(1-a_{0 l}\right)\right\} .
\end{aligned}
$$

Proof For the first objective function, it can be seen from Eqs. (7) and (10) that

$$
Z_{0 k}=\max \left\{0, \delta-\sum_{j=1}^{|I|}\left(a_{0 j}-a_{k j}\right) w_{j}\right\}
$$

if $w_{l}=1$ for an index $l$, then

$$
Z_{0 k}=\max \left\{0, \delta-\left(a_{0 l}-a_{k l}\right)\right\}
$$

$\forall l$, we can calculate the first objective function as:

$$
Q_{l}^{1}=\sum_{k=1}^{|I|} z_{0 l}
$$

then, the maximum value of the first objective function is

$$
Q_{\text {max }}^{1}=\max _{l}\left\{\sum_{k=1}^{|I|} \max \left\{0, \delta-\left(a_{0 l}-a_{k l}\right)\right\}\right\}
$$

Regarding the second objective function, if $w_{l}=1$ for an index $l$, then this equation (objective function) can be rewritten as:

$$
Q_{l}^{2}=\sum_{k=1}^{|I|}\left(1-a_{0 l}\right)
$$

then, the maximum value of the second objective function is 


$$
Q_{\max }^{2}=\max _{l}\left\{Q_{l}^{2}\right\}
$$

Acknowledgements This research was supported by the Universidad Panamericana through the grant "Fomento a la Investigación 2021 [grant number: UP-CI-2021-GDL-04-ING]."

Open Access This article is licensed under a Creative Commons Attribution 4.0 International License, which permits use, sharing, adaptation, distribution and reproduction in any medium or format, as long as you give appropriate credit to the original author(s) and the source, provide a link to the Creative Commons licence, and indicate if changes were made. The images or other third party material in this article are included in the article's Creative Commons licence, unless indicated otherwise in a credit line to the material. If material is not included in the article's Creative Commons licence and your intended use is not permitted by statutory regulation or exceeds the permitted use, you will need to obtain permission directly from the copyright holder. To view a copy of this licence, visit http://creativecommons.org/licen ses/by/4.0/.

\section{References}

Abastante F, Corrente S, Greco S, Ishizaka A, Lami IM (2019) A new parsimonious AHP methodology: assigning priorities to many objects by comparing pairwise few reference objects. Expert Syst Appl 127:109-120

Bandiera O, Barankay I, Rasul I (2009) Social connections and incentives in the workplace: evidence from personnel data. Econometrica 77(4):1047-1094. https://doi.org/10.3982/ECTA6496

Barron FH, Barrett BE (1996) Decision quality using ranked attribute weights. Manag Sci 42(11):1515-1523

Bateman MJ, Ludwig TD (2003) Managing distribution quality through an adapted incentive program with tiered goals and feedback. J Organ Behav Manag 23(1):33-55. https://doi.org/10.1300/ J075v23n01_03

Belton V, Stewart T (2002) Multiple criteria decision analysis: an integrated approach. Springer, New York

Bereketli I, Genevois ME, Albayrak YE, Ozyol M (2011) WEEE treatment strategies' evaluation using fuzzy LINMAP method. Expert Syst Appl 38(1):71-79. https://doi.org/10.1016/j.eswa. 2010.06.015

Cattin P, Punj G (1984) Factors influencing the selection of preference model form for continuous utility functions in conjoint analysis. Mark Sci 3(1):73-82

Cinelli M, Kadziński M, Gonzalez M, Słowiński R (2020) How to support the application of multiple criteria decision analysis? Let us start with a comprehensive taxonomy. Omega 96:102-261. https://doi.org/10.1016/j.omega.2020.102261

El Gibari S, Gómez T, Ruiz F (2019) Building composite indicators using multicriteria methods: a review. J Bus Econ 89(1):1-24. https://doi.org/10.1007/s11573-018-0902-z

Ertas N (2015) Turnover intentions and work motivations of millennial employees in federal service. Public Personnel Manag 44(3):401-423. https://doi.org/10.1177/0091026015588193

Gavish B, Gerdes JJH, Kalvenes J (2000) Performance-based reward distribution methods for anonymous decision-making groups. Group Decis Negot 9(5):393-413. https://doi.org/10.1023/A: 1008785931901

Giarlotta A (1998) Passive and active compensability multicriteria analysis (PACMAN). J Multi Criteria Decis Anal 7(4):204-216

Greco S, Figueira J, Ehrgott M (2016) Multiple criteria decision analysis: state of the art surveys. Springer, Berlin

Gunantara N (2018) A review of multi-objective optimization: methods and its applications. Cogent Eng 5(1):150-242. https://doi.org/10.1080/23311916.2018.1502242 
Holbrook MB (1981) Integrating compositional and decompositional analyses to represent the intervening role of perceptions in evaluative judgments. J Mark Res 18(1):13-28. https://doi.org/10. 2307/3151310

Horsky D, Nelson P (2006) Testing the statistical significance of linear programming estimators. Manag Sci 52(1):128-135

Horsky D, Rao M (1984) Estimation of attribute weights from preference comparisons. Manage Sci 30(7):801-822. https://doi.org/10.1287/mnsc.30.7.801

Julmi C (2019) When rational decision-making becomes irrational: a critical assessment and reconceptualization of intuition effectiveness. Bus Res 12(1):291-314. https://doi.org/10.1007/ s40685-019-0096-4

Kashef M, Safari H, Maleki M, Cruz-Machado V (2018) Solving MCDM problems based on combination of PACMAN and LINMAP. J Multi Criteria Decis Anal 25(5-6):169-176. https://doi.org/ 10.1002/mcda. 1650

Keeney RL, Raiffa H (1976) Decisions with multiple objectives: preferences and value trade-offs. Wiley, New York

Khairullah Z, Zionts S (1979) An experiment with some approaches for solving problems with multiple criteria. In: 3rd international conference on multiple criteria decision making, Konigswinter, Germany pp 20-24

Kim S (2018) Managing millennials' personal use of technology at work. Bus Horiz 61(2):261-270. https://doi.org/10.1016/j.bushor.2017.11.007

Kosfeld M, Neckermann S, Yang X (2016) The effects of financial and recognition incentives across work contexts: the role of meaning. Econ Inq 55(1):237-247. https://doi.org/10.1111/ecin.12350

Lai YL, Ishizaka A (2020) The application of multi-criteria decision analysis methods into talent identification process: a social psychological perspective. J Bus Res 109:637-647. https://doi. org/10.1016/j.jbusres.2019.08.027

Li DF (2008) Extension of the LINMAP for multiattribute decision making under Atanassov's intuitionistic fuzzy environment. Fuzzy Optim Decis Making 7(1):17-34. https://doi.org/10.1007/ s10700-007-9022-x

Linder S (2016) Fostering strategic renewal: monetary incentives, merit-based promotions, and engagement in autonomous strategic action. J Manag Control 27(2-3):251-280. https://doi.org/ 10.1007/s00187-015-0227-9

Marler RT, Arora JS (2005) Function-transformation methods for multi-objective optimization. Eng Optim 37(6):551-570. https://doi.org/10.1080/03052150500114289

Mausser H (2006) Normalization and other topics in multi-objective optimization. In: Proceedings of the Fields-MITACS Industrial Problems Workshop, Citeseer, pp 89-101

Milne P (2007) Motivation, incentives and organisational culture. J Knowl Manag 11(6):28-38. https://doi.org/10.1108/13673270710832145

Mullet GM, Karson MJ (1986) Percentiles of LINMAP conjoint indices of fit for various orthogonal arrays: a simulation study. J Mark Res 23(3):286-290. https://doi.org/10.1177/002224378602300 309

Nielsen (2015) Generational lifestyle survey. https://www.nielsen.com/wp-content/uploads/sites/3/ 2019/04/GlobalMillennialMindset.pdf

Pekelman D, Sen SK (1974) Mathematical programming models for the determination of attribute weights. Manag Sci 20(8):1217-1229

Rasouli S, Gharakhani M, Babakhani M (2011) A robust LINMAP for EFQM self assessment. Manag Sci Lett 1(2):213-222. https://doi.org/10.5267/j.ms1.2010.02.001

Russo JC, Dias MAG, da Silva B, Rocha A, Cyrino Oliveira FL (2018) Renegotiation in public-private partnerships: an incentive mechanism approach. Group Decis Negot 27(6):949-979. https:// doi.org/10.1007/s10726-018-9586-6

Saaty TL (1977) A scaling method for priorities in hierarchical structures. J Math Psychol 15(3):234-281

Sproten AN, Schwieren C (2015) Age differences in the reaction to incentives-a test of the successful ageing extension of social production functions theory. J Econ Ageing 6:176-186. https://doi. org/10.1016/j.jeoa.2015.02.002

Srinivasan V, Shocker AD (1973) Linear programming techniques for multidimensional analysis of preferences. Psychometrika 38(3):337-369. https://doi.org/10.1007/BF02291658

Stein G, Martin M (2015) Five keys to manage millennial talent: make way for a new generation. University of Navarra, IESE Business School, pp 1-8 
Tang J, Hsu T (2018) Utilizing the hierarchy structural fuzzy analytical network process model to evaluate critical elements of marketing strategic alliance development in mobile telecommunication industry. Group Decis Negot 27:251-284. https://doi.org/10.1007/s10726-018-9554-1

Tzeng GH, Huang JJ (2011) Multiple attribute decision making: Methods and applications. Chapman and Hall/CRC, London. https://doi.org/10.1201/b11032

Velazquez MA, Claudio D, Ravindran AR (2010) Experiments in multiple criteria selection problems with multiple decision makers. Int J Oper Res 7(4):413-428. https://doi.org/10.1504/IJOR.2010. 032419

Wittink DR, Cattin P (1981) Alternative estimation methods for conjoint analysis: a Monte Carlo study. J Mark Res 18(1):101-106. https://doi.org/10.2307/3151320

Xia HC, Li DF, Zhou JY, Wang JM (2006) Fuzzy LINMAP method for multiattribute decision making under fuzzy environments. J Comput Syst Sci 72(4):741-759. https://doi.org/10.1016/j.jcss. 2005.11.001

Yousaf A, Yang H, Sanders K (2015) Effects of intrinsic and extrinsic motivation on task and contextual performance of Pakistani professionals. J Manag Psychol 30(2):133-150. https://doi.org/10. 1108/JMP-09-2012-0277

Zhang Cq, Lu Yc (2006) The reverse logistics evaluation based on KPCA-LINMAP model. In: 2006 international conference on machine learning and cybernetics. IEEE, pp 2531-2535. https://doi.org/ 10.1109/ICMLC.2006.258844

Publisher's Note Springer Nature remains neutral with regard to jurisdictional claims in published maps and institutional affiliations.

\section{Authors and Affiliations}

\section{Jessica Rubiano-Moreno ${ }^{1} \cdot$ Samuel Nucamendi-Guillén $^{2}$ (I) Alvaro Cordero-Franco ${ }^{3}$. Alejandro Rodríguez-Magaña ${ }^{4}$}

Jessica Rubiano-Moreno

jesrubiano@udca.edu.co

Alvaro Cordero-Franco

alvaro.corderofr@uanl.edu.mx

Alejandro Rodríguez-Magaña

arodrigu@up.edu.mx

1 Facultad de Ciencias Administrativas y Comerciales, Universidad de Ciencias Aplicadas y Ambientales, Calle 222 \# 55 - 37, Bogotá, Colombia

2 Facultad de Ingeniería, Universidad Panamericana, Álvaro del Portillo 49, 45010 Zapopan, Jalisco, Mexico

3 Universidad Autónoma de Nuevo León, Facultad de Ciencias Físico Matemáticas, Av. Universidad s/n, 66451 San Nicolás de los Garza, Nuevo León, Mexico

4 Escuela de Ciencias Económicas y Empresariales, Universidad Panamericana, Álvaro del Portillo 49, Ciudad Granja, Zapopan, Jalisco, Mexico 